ID: 69

\title{
ULTRASOUND-GUIDED FEMORAL NERVE BLOCK AS A DIAGNOSTIC AID IN DEMONSTRATING QUADRICEPS INVOLVEMENT IN BOVINE SPASTIC PARESIS.
}

C. De Vlamynck1, L.Vlaminck1, S.Hauspie2, J.Saunders2, F.Gasthuys1

${ }_{1}$ Department of Surgery and Anaesthesiology of Domestic Animals, Faculty of Veterinary Medicine, Ghent University, Merelbeke, Belgium 2 Department of Medical Imaging of Domestic Animals, Faculty of Veterinary Medicine, Ghent University, Merelbeke, Belgium

Topic: 3. Bovine Medicine / Claw and Leg Diseases

The aim of this study was to evaluate the clinical effects of diagnostic anaesthesia of the femoral nerve performing a dorsal paralumbar injection technique in healthy calves and calves suffering from bovine spastic paresis. Based on bony landmarks and with ultrasound guidance, the femoral nerves of eight healthy calves were blocked with a $4 \%$ procaine solution containing blue dye. After euthanasia of the calves successful location of the injection was confirmed during dissection work. In 69 percent of the cases a paralysis effect of the quadriceps muscle was obtained after performance of the injection technique. A total paralysis of the quadriceps muscle was obtained in $50 \%$ of the cases. In $75 \%$ of the cases, the blue dye was less than $2 \mathrm{~mm}$ perineurally. Clinical use of the technique is demonstrated in two clinical cases suffering from atypical presentations of bovine spastic paresis. In calves suffering from these presentations of bovine spastic paresis an objective diagnostic tool is needed to declare an animal suitable for surgery to avoid unwanted aggravation of symptoms following partial tibial neurectomy. Femoral nerve blocking has the potential to be a valid diagnostic method to establish involvement of the quadriceps femoris muscles in young calves suffering from the quadriceps form (BSP-Q) or mixed presentation (BSP-M) of bovine spastic paresis 